\title{
Necesidades y características académicas laborales de los docentes que enseñan a investigar en la Facultad de Odontología de la UNAH, durante el tercer periodo de 2014
}

\author{
Santos Rómulo Barahona Cruz ${ }^{1}$ \\ Gustavo Torres ${ }^{2}$
}

\section{RESUMEN}

Las funciones de docencia e investigación son parte esencial de las estrategias que los sistemas de educación superior (ES) deben reorientar en los planes operativos a corto plazo (Oficina de Cooperación Universitaria, OC/U, 2010). Este artículo aborda desde la perspectiva técnica docente del autor, la enseñanza de la investigación en la Facultad de Odontología de la UNAH, durante el tercer período del año 2014.

El estudio permitió identificar características académicas y laborales de los docentes, describir necesidades sentidas y prescritas, así como hacer recomendaciones sobre el proceso de enseñanza-aprendizaje; para tal efecto, se seleccionaron 129 estudiantes de la clase de Métodos de investigación y sus respectivos docentes, cuyas edades oscilan entre 39 y 54 años, a quienes se les aplicó un cuestionario. Las bases de datos en SPSS-17.0 posibilitaron el análisis descriptivo con distribuciones de frecuencia y medidas de tendencia central.

Los resultados de la investigación revelan que el $67 \%$ de los estudiantes encuestados considera que los docentes realizan clases magistrales, el 18\% considera que utilizan la repetición o memorización, el 16\% dictados, el 30\% investigación acción, el $29 \%$ observación-análisis-reflexión, el $28 \%$ estudios de caso, el $71 \%$ trabajos en grupo, el $2 \%$ juego de roles, el $8 \%$ técnicas de resolución de problemas, el $17 \%$ debates, el $16 \%$ proyectos y el $8 \%$ foros.

Solo en uno de los docentes el nivel de conocimiento y manejo de la temática es alto.

\footnotetext{
1 Beneficiario de una beca de estudiante de posgrado de la Dirección de Investigación Científica y Posgrado, Maestría en Investigación Económica y Social, UNAH:rbarahonacruz@yahoo.es

2 Profesor universitario y asesor, Maestría en Investigación Económica y Social, Facultad de Ciencias Económicas y Contables, UNAH: gtorres_ddd@yahoo.com
} 
El nivel de logro alcanzado por los estudiantes en cada una de las competencias investigativas evaluadas, según los docentes, es bajo. El mínimo necesario, en cuanto a metodología, es la proyección de imágenes fijas como el recurso más utilizado (64\%) según opinión de los estudiantes y el uso de rotafolios es el menos utilizado (2 \%), lo que refleja el apego a métodos tradicionales de enseñanza.

Una vez determinadas las características laborales de los docentes, la conclusión es que se antepone la docencia a la investigación y el mismo plan de estudios de la carrera no responde a la necesidad de formar profesionales investigadores.

Palabras clave: enseñanza, aprendizaje, investigación, necesidades prescritas, características académicas, características laborales.

\section{ABSTRACT}

The functions of teaching and research are essential parts of the strategies in higher education systems (ES) that must shift short-term operational plans, OCU (2010). This article is based from the teaching and technical perspective of the author about the teaching of research in the Faculty of Dentistry at the UNAH during the third quarter of 2014. The study provides academic and occupational characteristics of teachers, describes needs, and makes recommendations regarding the process of learning; for this purpose, 129 students enrolled in the Research Methods class, as well as their teachers were selected as the sample. They were all given a questionnaire. The databases in SPSS-17.0 enabled descriptive analysis with frequency distributions and measures of central tendencies.

According to the students, $67 \%$ considered that teachers are practicing a method of teaching with explanations, $18 \%$ believe that they are using repetition and memorization, $16 \%$ believed that they are simply lecturing to the students, $30 \%$ teach by performing research, and $29 \%$ through observation-analysis-critical thinking, $28 \%$ teach through case studies, $71 \%$ by working in a groups, $2 \%$ throughout using role play, $8 \%$ through solving problems, $17 \%$ through debates, $16 \%$ through projects, and $8 \%$ through forums. Only one of the teachers in this study indicated a high level of knowledge and management of the subject. The level attained by students in each of the investigative skills assessed by teachers is low and is only the bare minimum. According to the students, in terms of methodology, the projection of still images is the 
resource most used by teachers (64\%) and flipcharts are the least used (2\%). Based on this, their process showed adherence to traditional teaching methods. Once the conclusions regarding the Curricular Plan of research were determined, it showed that the occupational characteristics of teachers and teaching in the Faculty did not respond to the need to train researchers in a professional manner.

Keywords: teaching, learning, research, prescribed needs, teacher academic characteristics, teacher occupational characteristics. 


\section{INTRODUCCIÓN}

La educación universitaria es, en su conjunto, un cúmulo de experiencias que preparan al individuo para la vida productiva y a la vez forma a las nuevas generaciones de docentes del nivel superior o expertos en determinado campo que seguramente realizarán algún aporte a la ciencia. Dicho proceso ha venido mejorando con el paso de los años, pues ya no se habla de experiencias de aprendizaje sin considerar los espacios pedagógicos abiertos; hoy en día se habla de internacionalización, adquisición de otras competencias y habilidades aparte de los conocimientos, así como de investigación cooperativa.

La Universidad Nacional Autónoma de Honduras (UNAH) es la principal institución de donde emanan los investigadores y los proyectos de investigación que servirán como inspiración para otros investigadores, pero también para quienes fungen como tomadores de decisiones en la solución de problemas que afectan a la sociedad en general. En tal sentido, el presente estudio sobre las necesidades y características académicas laborales de los docentes que enseñan investigación en la Facultad de Odontología de la UNAH, durante el tercer período 2014, es un primer diagnóstico sobre el impacto que tiene la investigación universitaria como medio de extensión hacia la sociedad en un campo específico, como la educación.

Así, esta investigación constituye parte de las iniciativas apoyadas por la Dirección de Investigación Científica y Posgrado (DICYP) de la UNAH, en el marco del proyecto: La enseñanza de la investigación en la educación superior pública en Honduras.

Indagar sobre las prácticas pedagógicas, las necesidades sentidas y el perfil del docente actual de la UNAH vinculado a la enseñanza de la investigación, permite evaluar qué tan distante o cercano se está del nuevo modelo educativo. En consecuencia, a partir de los resultados obtenidos, la UNAH podrá intervenir con propuestas de solución que conlleven a superar las debilidades que se han identificado, asimismo promover y dar sostenibilidad a las fortalezas con que ya se cuenta en el proceso de formación académica en investigación, del que son protagonistas los docentes.

La población beneficiaria de este proyecto es, en primera instancia, la constituida por los docentes universitarios, pues con la línea base se posibilita la concreción de programas y proyectos que vayan encaminados a ampliar su formación como 
docente universitario que imparte asignaturas de investigación; en segundo lugar, los estudiantes que cursan asignaturas de investigación quienes recibirán en un futuro cercano un efecto multiplicador de la capacitación docente. En términos globales, la UNAH como institución es la mayor beneficiaria, ya que el estudio se desarrolló con miras a mejorar la calidad de la educación universitaria en el área de investigación.

En general, el proceso de investigación fue exitoso y se llevó a cabo tal y como fue planificado con las autoridades de la Facultad, sin embargo, lo concerniente a la longitud de la encuesta fue el obstáculo más relevante debido a que el estudio, en general, buscaba obtener información relacionada con otros objetivos ajenos al tema aquí expuesto; dicho inconveniente se superó explicando a los participantes la dimensión del estudio.

\section{MÉTODO}

\section{Diseño}

La presente investigación se desarrolló con un diseño no experimental, ya que no se manipularon variables y no se construyó ninguna situación, sino que se observaron situaciones ya existentes. Atendiendo al criterio de Hernández, Fernández y Baptista (2006), la metodología a seguir en este estudio se planteó desde un enfoque cuantitativo con un alcance descriptivo, en donde se buscaba analizar las necesidades y características académicas laborales de los docentes de la Facultad de Odontología que forman parte del proceso de enseñanza aprendizaje de la investigación al impartir la asignatura de Métodos de investigación. Este trabajo, al ser una investigación que forma parte de un estudio general de cobertura nacional en los diferentes centros regionales de la UNAH, permite de forma práctica la comparación entre estudios similares, tal como lo explican Hernández y otros (2006).

Determinar las características académicas y laborales de los docentes y establecer las necesidades sentidas en relación con la enseñanza-aprendizaje de la investigación son los objetivos generales establecidos por la DICYP, a partir de los cuales un grupo de 4 investigadores planteó los objetivos específicos que se detallan a continuación: 
1. Identificar las características académicas de los docentes que forman parte del proceso de enseñanza- aprendizaje de la investigación en la Facultad de Odontología de la UNAH.

2. Enlistar las características laborales de los docentes que forman parte del proceso de enseñanza-aprendizaje de la investigación en la Facultad de Odontología de la UNAH.

3. Describir las necesidades sentidas con base a las competencias investigativas de los docentes en el proceso de enseñanza aprendizaje de la investigación en la Facultad de Odontología de la UNAH.

4. Identificar las necesidades prescritas en base al programa curricular de los docentes que forman parte del proceso de enseñanza-aprendizaje de la investigación en la Facultad de Odontología de la UNAH.

5. Enumerar algunas recomendaciones sobre la enseñanza aprendizaje de la investigación en la Facultad de Odontología de la UNAH.

A partir de estos objetivos el equipo de investigadores diseñó el instrumento de investigación, el cual se trató de un cuestionario compuesto de 94 preguntas cerradas con opciones de respuesta que fueron delimitadas previamente, algunas dicotómicas y otras con varias opciones de respuesta. La estructura del mismo para los estudiantes se constituyó en seis secciones: datos generales, características académicas, metodología de enseñanza-aprendizaje, formación en investigación, percepciones sobre el proceso de enseñanza-aprendizaje y competencias en investigación.

El cuestionario para los docentes incluyó las secciones anteriores, más una denominada: características laborales. Las preguntas relacionadas a la sección Competencias en investigación se organizaron bajo la modalidad de cuestionamientos múltiples, incluyendo una "batería de preguntas" Corbetta (2003); cuyas opciones de respuesta van de 0 a 4 en el nivel alcanzado, así: $0=$ no desarrollada, 1 = insatisfactorio, 2 = mínimo necesario, 3 = bueno, 4 = alto. Dicho instrumento fue auto administrado de forma individual en el caso de los docentes y de forma grupal en el caso de los estudiantes. 


\section{Población}

Para la realización de este estudio se consideran dos tipos de informantes: los docentes que imparten la asignatura Métodos de investigación y los estudiantes, quienes recibieron durante el tercer periodo académico del año en curso la asignatura denominada Métodos de investigación en la Facultad de Odontología. Se trabajó de la siguiente manera con los informantes del estudio:

1. Se tomó el universo de los docentes que imparten la asignatura Métodos de investigación en el último trimestre del periodo académico, de la Carrera de Odontología, a los que se aplicó un cuestionario.

2. En el caso de los estudiantes se trabajó con una muestra probabilística obtenida de los matriculados en dicha asignatura en el tercer periodo de la Carrera de Odontología, a los que se les aplicó un cuestionario.

Finalmente, la muestra la constituyeron 129 estudiantes (hombres y mujeres) de la Carrera de Odontología de la UNAH en Tegucigalpa, que cursan la asignatura de Métodos de investigación y 3 docentes que imparten dicha materia. Tanto estudiantes como docentes participaron en el estudio de forma voluntaria. La muestra es más que representativa del universo, ya que se trabajó con toda la población objeto de estudio.

\section{Entorno}

El presente estudio se llevó a cabo en la Facultad de Odontología, que es una dependencia de la UNAH en el área de ciencias biológicas y de la salud, en esta se forman profesionales de la Odontología con competencias y valores capaces de contribuir a la solución de problemas de salud bucal en el país. El plan de estudios ha sufrido algunas reformas y en el año 2000 fue readecuado y se mantiene hasta la actualidad, consta de 64 asignaturas con 288 unidades valorativas y un año de servicio social.

\section{Intervenciones}

Una vez estructurado el cuestionario se realizó la valoración por parte de los diferentes grupos que participaron en la investigación a nivel nacional, atribuyéndole los grados de pertinencia y adecuación a cada instrumento; después se le hicieron los cambios que se consideraron oportunos de implementar. El nuevo instrumento pasó 
nuevamente a ser valorado por el grupo de investigadores que participaron en la investigación a nivel nacional, así como una revisión por el asesor temático asignado. En este paso se contó con la revisión de una experta reconocida en el tema de la investigación universitaria, quien aportó información valiosa para tener un documento que proporcionara datos con los cuales se pudiera precisar mejor la información.

La prueba de confiabilidad se realizó mediante el método estadístico alfa de Cronbach y la prueba piloto del instrumento se realizó a través de un muestreo intencional, seleccionando a docentes que tuvieran conocimientos sobre la metodología de investigación, pero que no fuesen del universo de este estudio, para no contaminarlo.

\section{Análisis estadístico}

Los instrumentos recolectados se organizaron en dos bases de datos (una de docentes y otra de estudiantes), haciendo uso del software SPSS versión 17.0, a través del cual se obtuvieron tablas y gráficas que permitieron analizar los resultados obtenidos en base a los objetivos generales de la investigación; es decir, que para efectos de este estudio no solo se realizó el análisis considerando los objetivos específicos del tema de investigación particular en la Facultad de Odontología, sino que también se hizo conforme a los objetivos planteados en la propuesta inicial de la DICYP; de este modo, se llevó a cabo el análisis descriptivo de las variables (frecuencia y porcentajes). Los datos fueron analizados, principalmente, mediante las técnicas de la estadística descriptiva; en tal sentido, se utilizaron distribuciones de frecuencia (absoluta y relativa) y medidas de tendencia central (media y desviación estándar).

\section{RESULTADOS}

1. Perfil del estudiante de Odontología que cursa Métodos de investigación: los datos obtenidos revelan que 93 de los estudiantes encuestados son mujeres y 35 son hombres. La edad oscila entre los 19 a 39 años, siendo la frecuencia más alta la edad de 21 años (28 estudiantes), seguida del grupo con 20 años de edad (26 estudiantes). La frecuencia más baja corresponde a estudiantes con 34,35 y 39 años de edad (1 estudiante). Un 94 \% (120 estudiantes) de los encuestados son solteros y el $6 \%$ restante están casados, en unión libre o tienen una relación de pareja. 
2. Características académicas de los estudiantes: el $81 \%$ de los entrevistados posee título de bachillerato, el $8 \%$ son bachilleres técnicos, el $5 \%$ son peritos mercantiles y el $6 \%$ son maestros de educación primaria. El $60 \%$ estudió en un instituto privado y el $40 \%$ en un colegio público. Solo 3 de los estudiantes encuestados manifiestan tener nociones de investigación, porque ya cursaron Epidemiología, a diferencia de los restantes 124 estudiantes para quienes la asignatura Métodos de investigación es su primera y única experiencia de aprendizaje formal sobre investigación.

3. Metodología de enseñanza aprendizaje: el promedio de matriculados en la asignatura Métodos de investigación oscila entre 21 a 40 estudiantes, lo que coincide con el dato real proporcionado por los docentes en cada sección. De los estudiantes encuestados, el 98 \% (126) considera que el programa de la clase se ajusta a las necesidades de formación en investigación. La frecuencia más alta indica que prevalece el primer día de clases como el momento en que los profesores muestran el sílabo y el menos frecuente es aquellos que consideran que los profesores no lo muestran. El gráfico 1 y la tabla 1 muestran el porcentaje de los recursos didácticos utilizados por los profesores. Un $36 \%$ considera que los docentes utilizan materiales impresos y fotocopias, un $2 \%$ rotafolios, un 64 $\%$ proyección de imágenes fijas, un 9\% materiales sonoros, un $39 \%$ materiales audiovisuales, un $9 \%$ software especializado, un $33 \%$ bibliotecas o bases de datos y un $6 \%$ otros recursos como libro de texto, pizarra y clase magistral. La proyección de imágenes fijas es el recurso más utilizado (64 \%) según su opinión y uso de rotafolios el menos utilizado (2\%).

En su mayoría, el libro de texto que refieren los estudiantes como fuente de consulta que guía el proceso de enseñanza-aprendizaje se titula Metodología de la investigación, administración, economía, humanidades y ciencias sociales, escrito por César Bernal. El porcentaje asignado a los criterios que cumple el libro de texto antes mencionado es: $46 \%$ accesibilidad, $11 \%$ precio, $38 \%$ temática, $19 \%$ didáctica, $18 \%$ presentación, $15 \%$ autor y $3 \%$ otros. El criterio que prevalece es la accesibilidad (46\%), seguido de la temática (38\%) y el criterio que menos cumple el texto es el precio (11\%).

El gráfico 2 muestra las actividades que realizan los profesores para enseñar a investigar. El $41 \%$ considera la clase magistral, el $79 \%$ el trabajo en grupo, el $82 \%$ la exposición de los estudiantes, el $33 \%$ la elaboración de anteproyecto, el $57 \%$ la revisión de trabajos de investigación, el $7 \%$ las prácticas de campo y el $2 \%$ otros. 
La actividad que utilizan los profesores con más frecuencia son las exposiciones de los estudiantes y la que menos utilizan son las prácticas de campo.

Gráfico 1. Recursos didácticos que utiliza el profesor para impartir la asignatura de investigación

Materiales impresos y fotocopias

Rotafolios

Proyección de imágenes fijas

Materiales sonoros: Radio, discos, CD
Materiales audiovisuales: Películas, documentales Software especializado

Bibliotecas o bases de datos digitales Otros

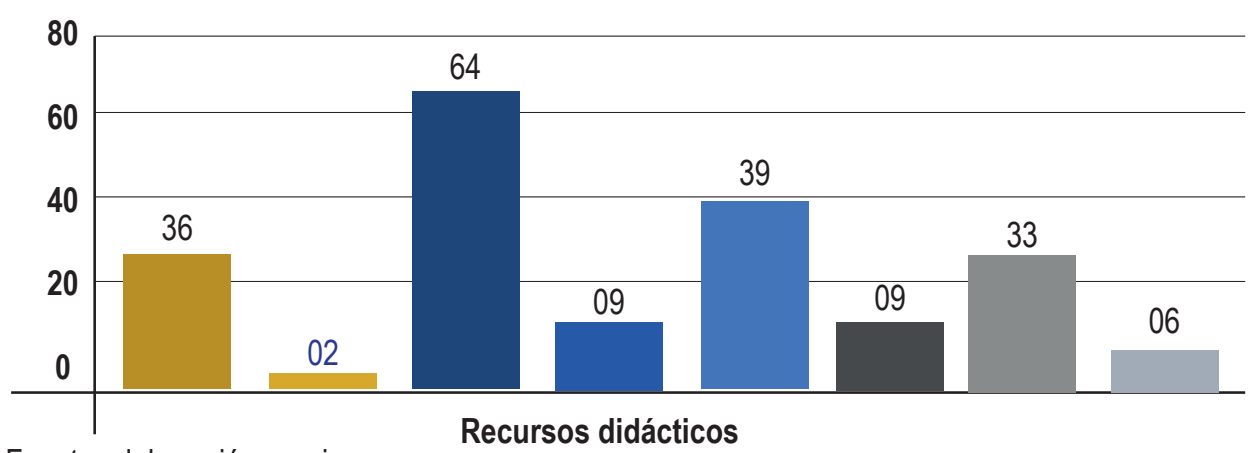

Fuente: elaboración propia.

Gráfico 2. Actividades que realiza el profesor para enseñar a investigar

Clase magistral

Trabajo en grupo

Exposición de los estudiantes

Elaboración de anteproyecto
Revisión de trabajos de investigación

Prácticas de campo

Otros

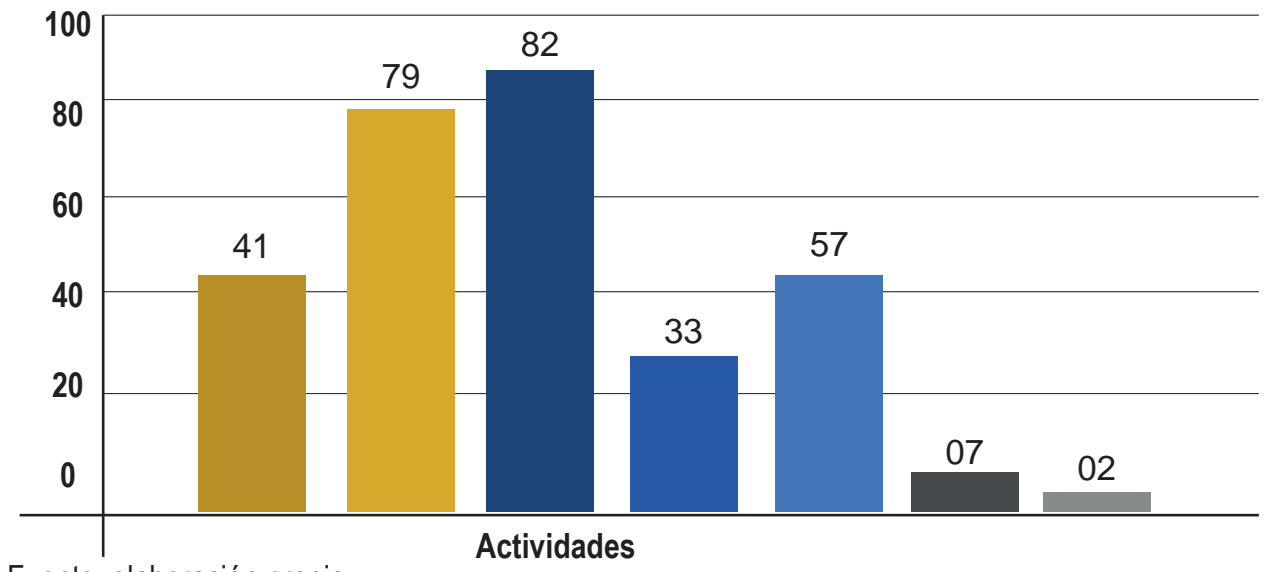

Fuente: elaboración propia. 
El gráfico 3 muestra de forma simultánea los mecanismos mediante los cuales el estudiante aprendió sobre técnicas cualitativas y cuantitativas de recolección de datos y los mecanismos que utilizó el docente para enseñar a presentar trabajos de investigación. El $28 \%$ de los estudiantes encuestados manifestó haber aprendido sobre técnicas cualitativas y cuantitativas de recolección de datos mediante lecturas, el $26 \%$ mediante exposiciones, el $77 \%$ mediante clases magistrales, el $5 \%$ mediante prácticas de campo y el $2 \%$ mediante otras formas como las tareas.

En cuanto a cómo enseñaron los docentes a presentar trabajos de investigación, el $93 \%$ considera que lo hizo mediante lecturas en revistas especializadas, tesis y resúmenes de investigación; el $33 \%$ por medio de exposiciones, el $57 \%$ a través de clases magistrales y el $2 \%$ con otras formas.

Gráfico 3. Mecanismos de enseñanza sobre técnicas de recolección de datos y presentación de trabajos de investigación

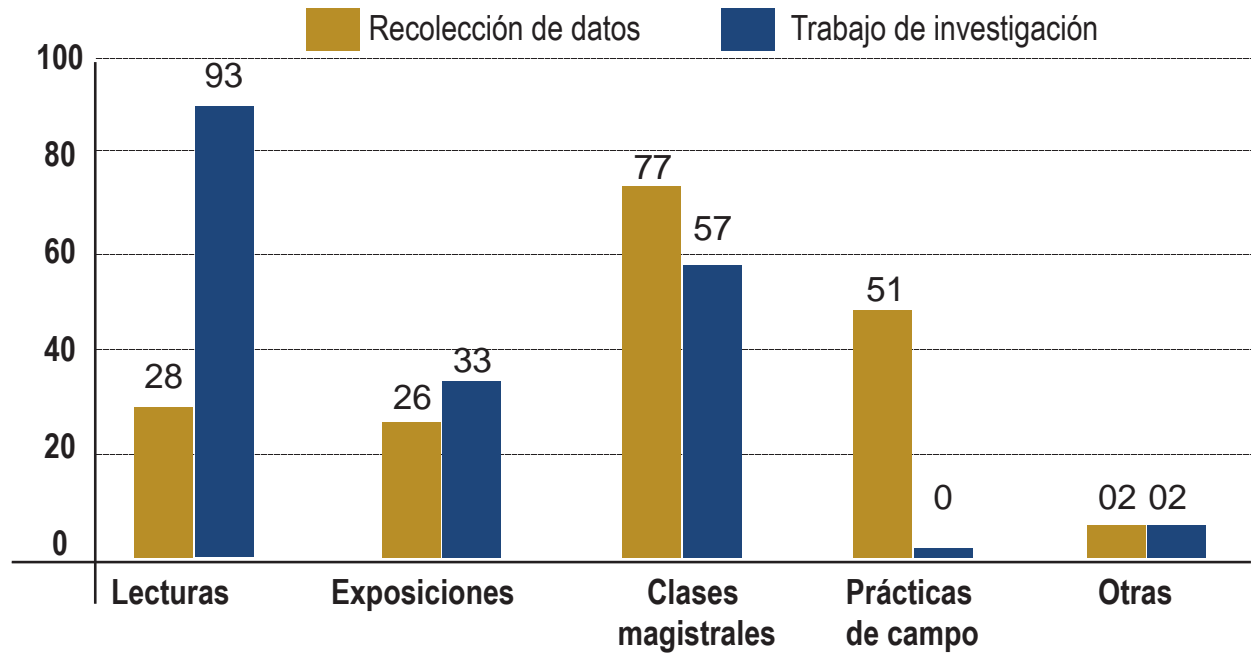

Fuente: elaboración propia. 
El gráfico 4 representa las actividades y técnicas que aplican los docentes en la clase de investigación. El $67 \%$ de los estudiantes encuestados considera que los docentes utilizan la clase magistral, el $18 \%$ considera que utilizan la repetición 0 memorización, el $16 \%$ dictados, el $30 \%$ investigación-acción, el $29 \%$ observación análisis-reflexión, el $28 \%$ estudio de caso, el $71 \%$ trabajos en grupo, el $2 \%$ juego de roles, el $8 \%$ técnicas de resolución de problemas, el $17 \%$ debates, el $16 \%$ proyectos, el $8 \%$ foros y el $2 \%$ otras.

En cuanto al proceso de evaluación, el $2 \%$ de los estudiantes encuestados considera que los docentes no toman sus expectativas y necesidades y es importante mencionar algunas razones como las siguientes: "al final, sino es tal cual ella dice, todo está malo; nos hace observar y comparar estudios, pero no a hacer los propios, lo que en mí es una mejor manera de aprender; se basa en lo que está el contenido de la clase".

4. Formación en investigación: con relación a la temática abordada por los docentes en la clase, más del $50 \%$ de los estudiantes reconoció los temas de investigación en general. Sin embargo, los temas que menos recuerdan son los relativos a enfoques de investigación, sistematización de información, operacionalización de variables o categorías de análisis, construcción del marco metodológico, muestreo, aplicación de instrumentos de investigación (cuestionarios, entrevistas, etc.), análisis de información recolectada, pruebas estadísticas, elaboración y presentación de informes de investigación.

De los temas enlistados, los porcentajes más altos de los estudiantes que no recuerdan haber estudiado dicha temática corresponden a las pruebas estadísticas (36\%) y el muestreo (28\%).

Con relación a las instancias de investigación de la UNAH que los estudiantes encuestados conocen y de las cuales reciben apoyo, el $73 \%$ conoce la existencia de la Dirección de Investigación Científica y Postgrado (DICYP), el $42 \%$ sabe de la existencia del Instituto de Investigaciones Económicas y Sociales (IIES), el $47 \%$ identifica al Instituto de Investigaciones Jurídicas (IIJ) y el 40\% reconoce al Instituto Universitario en Democracia, Paz y Seguridad (IUDPAS). 
Gráfico 4. Técnicas que aplica el profesor en el desarrollo de la clase de investigación
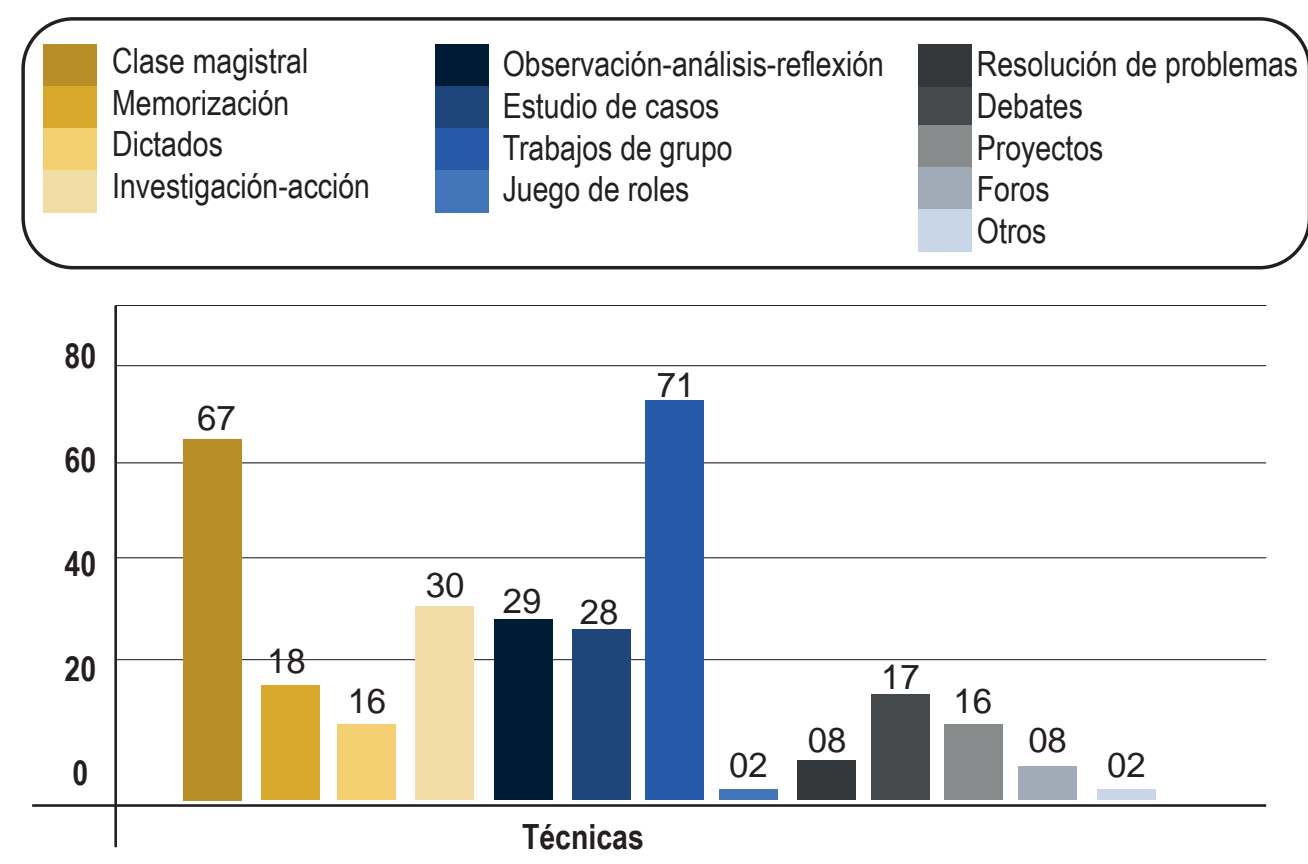

Fuente: elaboración propia.

5. Competencias obtenidas en investigación: el análisis de las competencias obtenidas como resultado de haber cursado la asignatura de Métodos de la investigación, se realizó de acuerdo con cinco competencias: capacidad de identificar problemas y formular preguntas, manejo metodológico, procesamiento de información, capacidad para comunicar resultados, responsabilidad y conducta ética. Los resultados obtenidos en cada una de ellas indica un nivel de logro "bueno" o el "mínimo necesario".

6. Perfil de los docentes que imparten la asignatura Métodos de investigación: los docentes encuestados fueron 2 mujeres de 51 y 54 años, respectivamente y 1 hombre de 39 años de edad. Dos de ellos están casados y uno es divorciado. Dos de los docentes son originarios de Tegucigalpa y el otro procede de Gracias, Lempira. Su carga académica solo difiere en el número de alumnos y secciones que atienden, dos de ellos tienen dos secciones con un total de 64 y 50 alumnos, respectivamente; a diferencia del otro docente que solo atiende una sección por la mañana con un total de 25 alumnos. 
7. Características académicas de los docentes: dos de los docentes poseen grado académico de maestría obtenido en universidades del extranjero (Nicaragua y México), a excepción del otro docente cuyo grado académico es de licenciatura. Uno de ellos ha recibido cuatro capacitaciones, a diferencia de los otros dos que solo han recibido dos. Los tres coinciden en haber recibido el Diplomado en Investigación Científica, dos han recibido el Diplomado en Enseñanza de la Educación Superior y el Curso Aprender. Únicamente uno ha recibido el Diplomado en Competencias. Ninguno de ellos ha recibido otras capacitaciones específicas en el área de investigación, sin embargo, dos de ellos pertenecen a organizaciones en las que se realiza investigación, tales como la Unidad de Gestión de la Investigación Científica, el Comité de Investigación y el Consejo de Investigación de la Facultad de Odontología.

8. Características laborales: dos de los docentes están en la categoría de profesor auxiliar y solo uno de ellos está en la categoría profesor titular II. Dos son docentes a tiempo completo y el otro se encuentra en la modalidad de profesor horario, ya que se le asignó un horario fuera de sus responsabilidades como administrativo. En cuanto a los años de servicio, sobresale uno con 27 años y 8 meses, a diferencia de los otros dos, quienes tienen entre dos a tres años y medio de servicio. Solo uno ha realizado proyectos de investigación en los últimos cinco años, de igual forma, dos de ellos han asesorado dos proyectos de investigación. Sin embargo, ninguno ha recibido algún tipo de reconocimiento relacionado con sus proyectos de investigación, porque no la han hecho. Los dos docentes que han formado parte de congresos de investigación ha sido como expositor y organizador, a diferencia del otro docente que solamente ha asistido en calidad de espectador.

Tabla 1. Recursos didácticos que utilizan los docentes en la asignatura

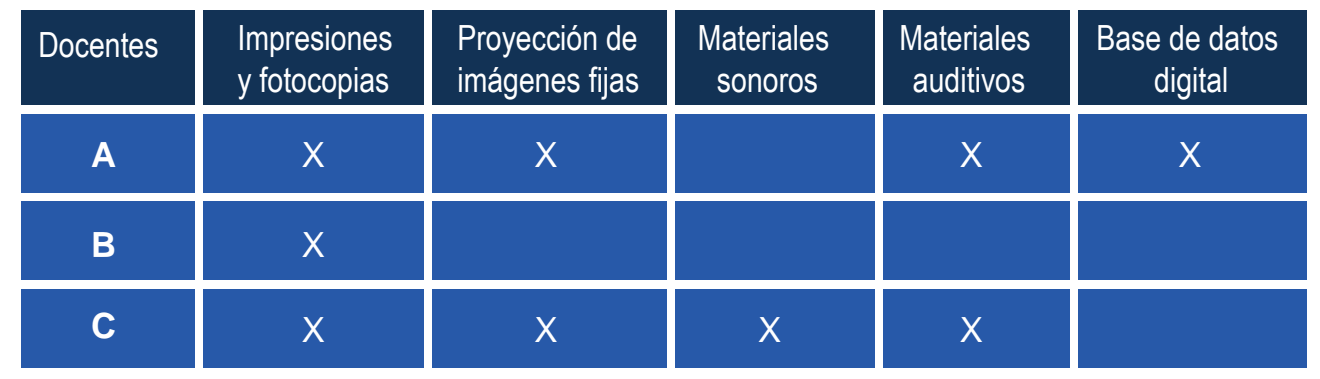

Fuente: elaboración propia. 
9. Según los docentes, la accesibilidad, didáctica, temática, autor y la facilidad de comprensión para el estudiante son los criterios por los que han elegido los textos que utilizan como fuente de consulta para planificar sus clases. El más utilizado es el de Bernal, sin embargo, utilizan otros como Investigación Científica en Ciencias de la Salud de B.P de Hungler y Denise Polit; Metodología de la investigación: Manual para el desarrollo de personal de salud de F.H. de Canales, E. L. de Alvarado y E.B. Pineda; Metodología de la investigación de R. H. Sampieri, C. F. Collado y P. B. Lucio.

10. Algunos aspectos que los docentes consideran importante para modificar sus planes de clase son los objetivos, el contenido, los métodos de enseñanza, los métodos de evaluación, los recursos didácticos, la duración del curso y las necesidades de los estudiantes. De estos, los más comunes fueron el método de evaluación, la duración del curso y las necesidades del estudiante; aunque dos de los docentes actualizan sus planes de clase cada trimestre y el otro lo hace cada año. Los tres coinciden en que el tiempo para cubrir el contenido planificado no es el adecuado, ya que hay temas que requieren de mayor explicación y deben ser abordados con mayor profundidad y lo que más afecta es que el alumno no tiene el tiempo suficiente para desarrollar un proyecto de investigación.

11. Para la elaboración del protocolo de investigación solo uno de los docentes usa como referente los temas prioritarios de la UNAH, sugiere bibliografía básica, asesora la selección del problema, redacción de objetivos y usa modelos o guías de investigación; a diferencia de los otros dos, quienes se limitan a asesorar la selección del problema o en el peor de los casos no elaboran protocolo de investigación. Dos de los docentes recurren a lecturas y clases magistrales para enseñar sobre técnicas de recolección de datos cualitativas y cuantitativas; a diferencia de uno de ellos que sumado a las anteriores usa exposiciones y prácticas de campo. De igual forma sucede con la instrucción sobre presentación de trabajos de investigación, dos de ellos se limitan a lecturas en revistas especializadas, resúmenes de investigación y clases magistrales; a diferencia del otro, que además de las anteriores recurre a lectura de tesis y exposiciones.

12. Formación en investigación: los tres docentes afirman impartir casi toda la temática concerniente a investigación, a excepción de la sistematización de la información, aplicación de instrumentos de investigación y pruebas estadísticas. El nivel de conocimiento y manejo de la temática en uno de los docentes 
es medio, en otro oscila entre medio y bajo, solo en uno de los docentes el nivel de conocimiento es alto en toda la temática. En la tabla 2 se observa el nivel de logro alcanzado por los estudiantes en cada una de las competencias evaluadas, según los docentes.

Tabla 2. Nivel de logro de las competencias adquiridas por los estudiantes en la asignatura de investigación

\begin{tabular}{|c|c|c|c|}
\hline \multirow{2}{*}{ Competencias investigativas } & \multicolumn{3}{|c|}{ Nivel alcanzado } \\
\hline & Docente A & Docente B & Docente C \\
\hline $\begin{array}{l}\text { Capacidad de identificar problemas y } \\
\text { formular preguntas }\end{array}$ & Bueno & Insatisfactorio & Mínimo necesario \\
\hline Manejo metodológico & Bueno & Bueno & Mínimo necesario \\
\hline \multicolumn{4}{|c|}{ Procesamiento de información } \\
\hline Recolección de información y datos & Mínimo necesario & Bueno & Bueno \\
\hline Manejo técnico & Mínimo necesario & No desarrollada & No desarrollada \\
\hline \multicolumn{4}{|c|}{ Capacidad para comunicar resultados } \\
\hline Escrita & Bueno & No desarrollada & No desarrollada \\
\hline Oral & Mínimo necesario & Insatisfactorio & No desarrollada \\
\hline Responsabilidad y conducta ética & Bueno & Bueno & Mínimo necesario \\
\hline Capacidad de trabajo en equipo & Bueno & Mínimo necesario & Mínimo necesario \\
\hline
\end{tabular}

Fuente: elaboración propia.

\section{DISCUSIÓN}

En esta muestra, la Carrera de Odontología es una opción académica que prevalece en estudiantes del género femenino, lo que podría ser consecuencia de patrones culturales inculcados socialmente, puesto que existe la creencia de que ciertas carreras no son para los hombres.

De acuerdo a la edad, se podría asociar que los estudiantes aún dependen económicamente de sus padres, ya que la mayoría son solteros. Un aspecto importante en cuanto a la frecuencia más baja es que se ha reducido la población estudiantil entre los 30 y 40 años de edad, lo que sugiere una buena aceptación de 
las reformas universitarias en cuanto al cumplimiento del plan de estudios en el tiempo establecido.

\section{Preparación en metodología de investigación}

El plan de estudios de la Carrera de Odontología carece de formación metodológica en investigación y no se está aprovechando al máximo el potencial de los futuros egresados de Odontología, en un campo que ofrece óptimas posibilidades para el desarrollo de la investigación científica. La Carrera de Odontología es una opción para estudiantes de un nivel socioeconómico medio, dado que el costo de los implementos que se requieren para los laboratorios y los libros de texto es alto.

Metodología de enseñanza-aprendizaje desde la perspectiva de los estudiantes

Los docentes que enseñan a investigar en la Facultad de Odontología desarrollan un programa tradicional de enseñanza y aún no implementan mecanismos de enseñanza activa como enviar el sílabo en formato digital vía correo electrónico. El uso de recursos didácticos denota el apego a una metodología tradicional, lo que implica que el docente no busca actualizar su método de enseñanza y la Facultad no cuenta con los recursos didácticos que exige la pedagogía moderna. El libro de texto que en su mayoría refieren los estudiantes como fuente de consulta que guía el proceso de enseñanza aprendizaje se titula Metodología de la investigación, administración, economía, humanidades y ciencias sociales, escrito por César Bernal; el cual es fácil de encontrar en el mercado, pero no está al alcance del presupuesto estudiantil.

Los comentarios acerca del tiempo para cubrir el contenido planificado por los profesores en la asignatura indican que el docente está desarrollando un método de enseñanza tradicional basado en la memorización y exige del alumno conocer el contenido desde una perspectiva teórica, dejando de lado la aplicación como nivel de destreza de pensamiento; además, el docente se interesa por cubrir los contenidos, pero no le da importancia al aprendizaje significativo de los estudiantes.

Prevalece asignar trabajo extra como estrategia adicional que utilizan los profesores si se interrumpe el desarrollo del contenido de la asignatura de investigación, de esta forma los alumnos no cuentan con la asesoría técnica metodológica para su ejecución o se enfrentan a lecturas recomendadas que no comprenden, ya que es un campo totalmente nuevo para el estudiante de esta Facultad. Esto refleja que existe 
un autoaprendizaje de conceptos teóricos sobre el tema y no se lleva a la práctica el proceso mismo de investigación.

La didáctica que utilizan los docentes en el proceso de enseñanza refleja falta de formación pedagógica, es decir, que los docentes tienen conocimientos teóricos, pero no han logrado desarrollar las competencias apropiadas para transmitir el conocimiento a los estudiantes de forma no tradicional. Con relación a la temática abordada por los docentes en la clase, la información obtenida corrobora la importancia que representa para el docente el abordar los contenidos coprogramados, sin considerar la calidad del aprendizaje y la longitud de cada uno de dichos contenidos; a su vez manifiesta la necesidad de dividir el contenido por lo menos en dos secciones, una parte relacionada con metodología y otra estrictamente con muestreo y análisis de información, ya que por la naturaleza del contenido se vuelve más complicado, incluso a nivel de posgrado.

Más del $50 \%$ de los estudiantes en esta muestra no tiene conocimiento de la existencia de todas las instancias de investigación en la Universidad, a excepción de la DICYP, esto seguramente se debe a que la mayoría de ellos cursa sus primeros años de estudio en la UNAH y la investigación propiamente dicha no fue un campo del cual tuvieran información previa como actividad ocupacional a nivel de secundaria. El conocimiento de la DICYP es por el papel que cumple dicha instancia como unidad principal de investigación en la UNAH, más la promoción a nivel estudiantil y docente de las actividades que realiza con el fin de promover la investigación en los últimos años.

Competencias obtenidas en investigación según los estudiantes

La asignatura de Investigación en la Facultad se imparte de forma prematura sin tener un fundamento básico previo, lo que explica el porqué el nivel de logro de la mayoría de las competencias es "bueno" o el "mínimo necesario", ya que los estudiantes aún no desarrollan habilidades en el campo de la investigación y menos todavía los conocimientos de su propio campo de formación en las ciencias de la salud. Lo más oportuno, en este aspecto, sería dividir el curso en dos grandes unidades, una dedicada a introducir los fundamentos básicos de metodología de la investigación y otra para poner en práctica la teoría sobre formación en investigación. El tiempo que se dedica a enseñar la materia es poco con relación a los contenidos establecidos en el sílabo, por lo que se abordan los temas de modo fugaz, sin dedicar tiempo de calidad a aprovechar la puesta en práctica de aquellas competencias que 
se prestan precisamente por el campo de trabajo de la odontología, adonde es más que factible realizar estudios científicos.

Sumado a esto, en la Facultad no se dispone de los recursos didácticos ni del personal docente idóneo para la enseñanza de la materia, debido a que no es una asignatura propia de la Carrera de Odontología, según afirma uno de los encuestados: "Los docentes evaden la responsabilidad de enseñar investigación, porque no les gusta" y es tan grave el caso que se ven en la necesidad de asignar uno de los cursos a un administrativo: "Quien hace su mejor esfuerzo por enseñar, pero no tiene la formación requerida para tal efecto".

Para los docentes que enseñan a investigar es casi imposible transmitir en un solo curso aquellas competencias que forman parte de otras áreas del saber y que son imprescindibles en el manejo de ciertos programas útiles para procesar información, elaborar gráficos y tablas, redactar de forma eficiente, buscar información haciendo uso de otros idiomas, etc. En la mayoría de los casos, estas competencias no fueron bien aprehendidas en la secundaria y de una u otra forma limita el hecho de que los docentes pudieran llevar a los estudiantes a un nivel práctico, es decir, que todo se queda a nivel informativo. Aquí se nota la falta de preparación en las cuestiones básicas para enfrentarse al proceso de investigación y, por ende, la necesidad de implementar la enseñanza de los fundamentos básicos de investigación en la escuela secundaria. Al igual que las competencias anteriores, en cuanto al manejo metodológico, el nivel de logro indica que falta hacer hincapié en ciertos temas que se prestan para abordarlos mediante la aplicación como nivel de destreza.

Las competencias obtenidas en la clase de investigación en cuanto a recolección de información y datos son propias de la asignatura de Español, por ello se espera que el nivel de logro sea alto, puesto que es una asignatura general, sin embargo, todo el panorama que aquí se observó podría mejorar al elaborar planes de clase de forma integrada buscando, por un lado, evitar la repetición de contenidos coprogramáticos $y$, por otro, encontrar el sentido utilitario a la materia preparando a los estudiantes en el manejo de competencias que les serán oportunos en sus trabajos de investigación.

Es interesante ver que aunque vivimos en un mundo tecnificado, el hecho de navegar en internet no significa que los estudiantes adquieran competencias útiles para la vida profesional y competitiva del mundo moderno, esto indica que los docentes al enseñar dan por sentado la adquisición de ciertas competencias en cuanto a manejo técnico y no es así. 
Los docentes de la Facultad deberían dedicarse a enseñar y a la vez producir resultados de investigación, sin embargo, hasta ahora los títulos y credenciales no dejan de ser requisitos para ostentar un cargo, porque no existen productos que midan su desempeño.

El hecho de que no se dediquen a investigar va unido a la búsqueda de actualización e innovación pedagógica, no obstante, no implementan nuevas estrategias de enseñanza o tratan de innovar sus procedimientos didácticos, ya que no se interesan en recibir otras capacitaciones que les permitan salir de lo tradicional en la enseñanza.

Al parecer, la asignatura Métodos de investigación ha sido de reciente incorporación al programa de estudios de la Facultad y el mismo personal docente no le ha brindado importancia a la materia, añade uno de los encuestados: "Ningún docente a tiempo completo ha querido enseñar esta asignatura". Todo parece indicar que el problema en cuanto a la didáctica de enseñanza no es algo que les afecte recientemente, sino que es más bien la causa del por qué en la Facultad no se haya dado énfasis a la investigación y, por ende, no existan producciones y publicaciones científicas.

\section{Metodología de enseñanza-aprendizaje desde la óptica de los docentes}

Con relación a la metodología de enseñanza, los docentes consideran que el programa de la clase no se ajusta a las necesidades de formación de los estudiantes en investigación debido a aspectos que ya han sido enunciados anteriormente, tales como disponibilidad de tiempo, amplitud de los contenidos, programa de estudios. Las justificaciones al respecto fueron: "En una sola clase no se abarca suficiente; se requiere de mayor tiempo para ampliar contenidos y la asignatura está en bloques que los estudiantes no han llevado clases profesionalistas", con esta última se refiere a que los estudiantes aún no tienen una base de conocimientos propios del campo de la Odontología para investigar haciendo uso de conceptos y con una terminología más especializada en general.

Con relación a las opciones que el docente considera en su planificación, adicional al programa de la clase entregado por la coordinación, figuran el Modelo Educativo de la UNAH, la retroalimentación de los exestudiantes y las experiencias adquiridas. En cuanto a este punto es importante mencionar que las observaciones que puedan hacer los exestudiantes se limitarán al manejo de la didáctica, ya que son estudiantes que aún no tienen el criterio para establecer comparaciones desde una perspectiva más amplia, por lo que se sugiere promover la supervisión de otras entidades e 
incluso de estudiantes de otro nivel académico que les permita trabajar en conjunto para mejorar los resultados.

En la elaboración del sílabo de la asignatura participa una subcomisión de desarrollo curricular y el mismo docente. De igual forma, es imprescindible que en esta etapa no solo participen expertos en currículo, sino expertos en investigación que puedan dimensionar los ajustes pertinentes para la enseñanza de los temas y la puesta en práctica de los mismos. Un estudio realizado en Colombia sobre leer textos científicos y académicos en la educación superior (Carlino, 2003), sostiene que los estudiantes al utilizar fotocopias en la universidad se enfrentan a obstáculos como falta de comprensión, la calidad de las duplicaciones es escasa, la visualización de lo impreso se dificulta, la lectura se realiza fuera del contexto con los capítulos precedentes 0 posteriores, sin conocer índices, prólogos, introducciones, sin solapas que presenten a sus autores, comentarios del texto $\mathrm{y}$ a veces sin referencias bibliográficas completas ni fechas de publicación; lo cual no permite al estudiante ubicarse dentro de lo que lee. Por estas y otras razones que se consideran en una estrategia de enseñanza activa, opuesta a la tradicional, el uso de impresiones y fotocopias pasó a ser algo obsoleto.

Con respecto a la metodología, predomina la forma tradicional, la que es evidente en todo el proceso didáctico, ya que predomina en el desarrollo de sus clases el apego a actividades como la clase magistral, repetición, memorización, trabajos en grupo y dictados. Las actividades menos frecuentes fueron investigación-acción, observación-análisis-reflexión, juego de roles y debates. El hecho de que los docentes no utilicen otras actividades para salir de la rutina, es porque requiere de tiempo para planificarlas y llevarlas a la práctica y como ya se ha expuesto, una limitante para ellos es el tiempo y la cantidad de contenido que figura en el sílabo.

\section{Percepciones sobre el proceso de enseñanza-aprendizaje}

Una deficiencia que los docentes encuentran en cuanto al proceso de enseñanza de la investigación en la Facultad, es que en el plan de estudios de la Carrera de Odontología no se aborda ampliamente la investigación y ello requiere que los docentes tengan amplios conocimientos sobre metodologías de investigación, de forma que están conscientes de que les falta preparación en el campo metodológico.

Dos de los docentes reconocen enseñar a investigar aunque no les gusta y esta puede ser una razón de peso que explique por qué el nivel alcanzado de muchas 
competencias de los estudiantes en investigación es el mínimo necesario o bueno, puesto que es complicado transmitir algo por lo que no se siente gusto o motivación; de hecho, los docentes afirmaron que los estudiantes no adquieren las competencias investigativas en su totalidad durante las clases de investigación, así, están conscientes de que la experiencia en investigación es fundamental para enseñar a investigar, ya que es importante predicar con el ejemplo; a pesar de que consideran tener amplios conocimientos en programas estadísticos, no los aplican en este campo. En conclusión, los tres docentes no se consideran docentes investigadores.

\section{Competencias de los estudiantes en investigación de acuerdo a los docentes}

En casi todas las competencias que ya se enunciaron en el apartado de los estudiantes, coincide el resultado obtenido mediante las respuestas brindadas por los docentes, quienes consideran que el nivel alcanzado por los alumnos como resultado de la clase de investigación es bueno y el mínimo necesario; en raras ocasiones en algunos aspectos implícitos en alguna competencia es alto, por lo que se deduce que existe una gran deficiencia en el logro de los objetivos de aprendizaje de la investigación y no se está llevando al estudiante a un nivel de exigencia óptimo en la materia. Esto concuerda con lo que dice Zelaya (2012) con relación al perfil de los estudiantes: "Honduras no ha contado con una política educativa de largo plazo que defina de manera clara y precisa el perfil de los (as) estudiantes. Esta falencia a la larga ha generado serias deficiencias en relación a las habilidades, destrezas y conocimientos que se deberían formar en cada nivel educativo" (p. 362).

\section{CONCLUSIONES}

1. Dentro de las características académicas se reconoce, posteriormente al estudio, la realización del docente al obtener un título universitario de posgrado a nivel de maestría, ya sea por iniciativa propia o por exigencia de la universidad, es decir, que son profesionales con una preparación en la rama de salud y servicios sociales según la clasificación de la UNESCO. Sin embargo, la formación académica en el mundo de la docencia, que por supuesto cuando está ligada a la investigación, no tiene un techo y debe ser integral en el amplio sentido de la palabra; según lo encontrado en los resultados de la investigación sobre la enseñanza de la investigación, los docentes de la Facultad de Odontología no tienen la suficiente capacitación tanto en temas de investigación como en metodología 
de la enseñanza. Esto permite visualizar la necesidad apremiante de la UNAH, como institución garante del desarrollo de la investigación como un todo, en mejorar los mecanismos de contratación de docentes que se dedicarán a enseñar investigación y, por supuesto, de gestionar de forma permanente su participación en capacitaciones que les permitan actualizar sus conocimientos en docencia e investigación.

2. En lo que concierne a las características laborales de los docentes, es importante resaltar que se antepone la docencia a la investigación, es decir, que los docentes realizan su labor o trabajo de enseñar investigación, pero no hacen investigación. No cabe duda que hay una estrecha relación entre las características académicas y laborales de los docentes, debido a que todas las capacitaciones recibidas, años de servicio y de hecho las investigaciones que realicen, se consideran requisitos fundamentales para posicionarse en determinada categoría docente, no obstante, en la Facultad de Odontología más del $50 \%$ de los docentes que enseñan a investigar son recientes en la docencia universitaria, no se dedican a investigar y, por ende, no realizan publicaciones científicas; por supuesto, esto se convierte en una limitante en el proceso metodológico de la asignatura de investigación, porque de alguna manera forma parte del factor motivacional del individuo en el campo profesional.

3. Al indagar sobre la metodología de enseñanza aprendizaje de la investigación que incluye planes y programas de estudio, sílabos o guías curriculares, recursos didácticos, técnicas o procedimientos didácticos y la evaluación, entre otros aspectos, además la formación específica de los docentes en investigación, las percepciones tanto de los docentes como de los estudiantes y la valoración de algunas competencias en investigación, permiten establecer las necesidades prescritas y sentidas de los docentes que enseñan a investigar en la Facultad de Odontología. En este contexto, dicha Facultad carece de un programa bien estructurado en formación integral de investigación y el mismo plan de estudios de la carrera no se ajusta a la necesidad de formar profesionales investigadores. Tomando como referencia el hecho de que el mercado laboral actual exige mayor preparación, la UNAH debe ajustar sus planes de estudio para lograr una formación educativa acorde a su función tripartita: docencia-investigación- extensión.

4. La Facultad de Odontología no cuenta con los recursos didácticos apropiados para la enseñanza de la investigación propiamente dicha, las condiciones de los 
salones de clase son deficientes y no se dispone del equipo necesario para salir de la rutina tradicionalista desde el punto de vista pedagógico. El personal docente tiene el deseo de transmitir los conocimientos de la mejor manera, pero falta apoyo técnico administrativo para tal efecto. De igual forma, se concluye que las técnicas, procedimientos, actividades y mecanismos de evaluación que utilizan los docentes evidencian el apego a una metodología estrictamente tradicional. Las reformas que surjan a partir de este estudio deberán abordarse de forma integral en el contexto metodológico en su conjunto.

5. Como entidad académica que coordina los procesos de investigación de la UNAH, la DICYP es percibida por los docentes que enseñan a investigar como un organismo que dicta las directrices para cumplir la función de la Universidad en cuanto a investigación, sin embargo, no se refieren a esta como una entidad que apoye a los docentes en sus necesidades académicas, conocer sus ideas, implementar sus proyectos, supervisar su buen desempeño, mejorar su perfil como investigadores, etc. $\mathrm{O}$ sea, todo aquello que contribuiría a que el docente se sienta investigador cumpliendo con los requerimientos establecidos para ostentar el cargo de docente que enseña a investigar en la Facultad, pero no se logra la realización plena del docente investigador que disfruta de hacer ciencia, produce nuevos conocimientos y los publica en congresos y otros medios.

\section{AGRADECIMIENTOS}

A la DICYP por la invitación a formar parte de la experiencia en investigación a través del proyecto colaborativo; es especial al máster Edwin Medina, por su colaboración en cada etapa del proceso. De igual forma se agradece a los alumnos de la Facultad de Odontología que participaron en el estudio y a los docentes que imparten la asignatura, particularmente a la doctora Carmen Gutiérrez, quien coordina la Unidad de Gestión de la Investigación Científica. 


\section{BIBLIOGRAFÍA}

Carlino, P. (2003). Leer textos científicos y académicos en la educación superior. Obstáculos y bienvenidas a una cultura nueva, 3 (2). Facultad de Educación, Universidad de Antioquia, Medellín, Colombia. Recuperado de: aprendeenlinea.udea.edu.co/revistas/index.../11146

Corbetta, P. (2003). Metodología y técnicas de investigación social. Madrid: McGraw-Hill.

Hernández, R.; Fernández, C. y Baptista, P. (2007). Metodología de la investigación. México: Editorial McGraw Hill.

Oficina de Cooperación Universitaria. (2010). Tendencias universidad 2020, estudio de prospectiva. Madrid:Gráficas Muriel.

Zelaya, M. (2012). En tres décadas de transición política en Honduras. Tegucigalpa: Editorial Universitaria. 